\title{
A new bidirectional intraperitoneal and systemic induction chemotherapy (BISIC) for the peritoneal metastasis from gastric cancer in neoadjuvant setting
}

Yonemura $\mathrm{Y}^{1,7 *}$, Canbay $\mathrm{E}^{1,8}$, Endou $\mathrm{Y}^{2}$, Ishibashi $\mathrm{H}^{1}$, Mizumoto $\mathrm{A}^{1}$, Li $\mathrm{Y}^{3}$, Liu $\mathrm{Y}^{3}$, Takeshita $\mathrm{K}^{1}$, Ichinose $\mathrm{M}^{1}$, Takao $\mathrm{N}^{1}$, Saitou $\mathrm{T}^{1}$, Noguchi $\mathrm{K}^{1}$, Hirano $\mathrm{M}^{1}$, Glehen $\mathrm{O}^{4}$, Brücher $\mathrm{B}^{5}$ and Sugarbaker $\mathrm{P}^{6}$

${ }^{1} \mathrm{NPO}$ to Support Peritoneal Surface Malignancy Treatment, Oosaka, Japan

${ }^{2}$ Department of Experimental Therapeutics, Cancer Research Institute, Kanazawa University, Japan,

${ }^{3}$ Department of Surgery, Wuhan University, China

${ }^{4}$ Departement de Chirurgie Gēnerale, Centre Hospitalier Lyon-Sud Hospices Civils de Lyon, Universitē Lyon, France

${ }^{5}$ Surgical Oncology, Department of Surgery, Túbingen Comprehensive Cancer center, University of Túbingen, Germany

${ }^{6}$ Center of Gastrointestinal Malignancies, Program in Peritoneal Surface Malignancies, MedStar Washington Hospital Center, USA

${ }^{7}$ Department of Regional Cancer Therapies, Peritoneal Surface Malignancy Center, Kishiwada Tokusyukai Houspital, Kusatsu General Hospital, Japan

${ }^{8}$ Department of General Surgery, Kocaeli-Drince, Education and research Hospital, Istanbul, Turkey

\begin{abstract}
A phase II study to evaluate the efficacy and safety of a Bidirectional intraperitoneal and systemic induction chemotherapy (BISIC) were performed in patients with Peritoneal metastasis (PM) from gastric cancer in neoadjuvant setting. Sixty-one patients were treated with oral administration of S1 (60 mg/m²/day) for 14 consecutive days, followed by 7 days rest, plus intraperitoneal (i.p.) and intravascular (i.v.) administration of docetaxel and cisplatin $\left(30 \mathrm{mg} / \mathrm{m}^{2}\right.$ each) on day $1 \mathrm{and}$ on day 8. The treatment course was repeated every 3 weeks for 3 times. Positive cytological results in 38 patients before BISIC became negative in 27 (71.1\%) patients after BISIC. After BISIC, 44 patients received laparotomy and CRS, and complete cytoreduction was achieved in 28 of 44 patients (64\%). During BISIC, side effects of grade 3 and 4 were found in $6(9.9 \%)$ patients. After CRS, $7(15.8 \%)$ and $3(6.8 \%)$ patients developed Grade 3 and 4 complications. The overall operative mortality rate was $4.5 \%$ (2/44). Histologic effects on primary tumors were found in $87.9 \%$ (29/33 tumors). Complete histologic disappearance of PM was observed in $10(22.7 \%)$ of 44 patients. Median survival time (MST) was 15.1 months, with a one and two-year survival of $62.4 \%$, and $44.0 \%$. BISIC therapy is safe and effective in gastric cancer patients with PM.
\end{abstract}

\section{Introduction}

The prognosis of gastric cancer patients with peritoneal metastases $(\mathrm{PM})$ is extremely poor. Systemic chemotherapy or Cytoreductive surgery (CRS) alone does not improve the long-term survival of patients with PM. All the patients died of disease within 8 years after systemic chemotherapy with or without gastrectomy [1]. During recent two decades, a new therapeutic approach based on the combination of Cytoreductive surgery (CRS) and Perioperative intraperitoneal chemotherapy (PIC) has been developed [2,3]. During operation, the macroscopic disease is completely removed by the peritonectomy techniques and the residual micrometastases are treated with Hyperthermic intraoperative intraperitoneal chemotherapy (HIPEC). Complete removal of primary tumor, regional lymph nodes and PM is essential for the long-term survival. Unfortunately, complete removal of PM is sometimes difficult when patients have diffuse involvement of the peritoneum. Accordingly, neoadjuvant intraperitoneal chemotherapy was developed to diminish PM before CRS [4]. Yonemura et al. reported the results Neoadjuvant intraperitoneal/ systemic chemotherapy (NIPS), and patients with PM was treated by systemic chemotherapy of S-1 and intraperitoneal (i.p.) docetaxxel and cisplatin. After treatment by NIPS, the rate of complete cytoreduction increased, and allover survival after NIPS in combination with CRS was improved [5]. However, the results after NIPS are still dismal.

Recently, we developed a combination chemotherapy named Bidirectional intraperitoneal and systemic induction chemotherapy
(BISIC) for the PM from gastric cancer in neoadjuvant setting. The regimen consists of i.p. and i.v. docetaxel and cisplatin once a 3 week interval to daily systemic chemotherapy of S-1 for 14 days. The present study demonstrated the results of a phase II clinical trial of BISIC to evaluate the efficacy and tolerability in gastric cancer patients with PM.

\section{Patients and methods}

The eligibility criteria were as follows: histological proven primary and recurrent gastric cancer with PM, age younger than 75 years old, Eastern Cooperative Oncology Group performance status of zero to two, adequate bone marrow, liver, and renal function, and expected survival period of longer than 3 months. Patients were excluded if they had metastasis to distant organ sites, other active concomitant malignancies, or other severe medical conditions.

Correspondence to: Yutaka Yonemura, MD, PhD, Director, NPO Organization to Support Peritoneal Surface Malignancy Treatment, Oosaka, Japan and Department of Regional Cancer Therapies, Peritoneal Metastasis Center, Kishiwada Tokusyukai Houspital, Kusatsu General Hospital, Shiga, Japan, E-mail: y.yonemura@coda.ocn.ne.jp

Key words: chemotherapy, BISIC, peritoneal metastasis, gastric cancer, neoadjuvant setting

Received: September 24, 2014; Accepted: October 03, 2014; Published: October 07,2014 
Yutaka Yonemura (2014) A new bidirectional intraperitoneal and systemic induction chemotherapy (BISIC) for the peritoneal metastasis from gastric cancer in neoadjuvant setting

Written informed consent was obtained from all patients. This study was carried out in accordance with the Declaration of Helsinki. This study was approved by the Ehics Committee of Kishiwada Tokushukai Hospital, with ethical approval number H19. Sixty-one patients who were newly diagnosed as advanced gastric cancer or recurrent gastric cancer were enrolled in this study when PM was histologically confirmed.

A peritoneal port system (Hickman Subcutaneous port; BARD, Salt Lake City, UT, USA) was introduced into the abdominal cavity under local anesthesia, and the tip was placed on the cul-de-sac. Then, a peritoneal wash cytology was performed after $500 \mathrm{ml}$ of physiological saline was injected into the peritoneal cavity. Papanicolaou staining was done before and at the end of each course.

S1 was administered orally twice daily at a dose of $60 \mathrm{mg} / \mathrm{m}^{2} /$ day for 14 consecutive days, followed by 7 days rest. Docetaxel and cisplatin were administered i.p. at a dose of $30 \mathrm{mg} / \mathrm{m}^{2}$ on day 1 . Docetaxel and cicplatin was diluted in $500 \mathrm{ml}$ of normal saline and administered through the implanted peritoneal port system. The same dose of docetaxel and cisplatin were administered i.v. on day 8 after standard premedication. The treatment course was repeated every 3 weeks for 3 times.

\section{Selection criteria of patients for cytoreductive surgery (CRS) after BISIC}

After three cycles of BISIC, patients who had the following criteria are excluded as the candidates for CRS: 1) evidence of para-aortic lymph node involvement and distant hematogenous metastasis confirmed by Computed tomography (CT), or Magnetic resonance imaging (MRI), 2) patients with progressive disease after BISIC or 3) patients with severe co-morbidities or poor general condition.

\section{Evaluation of tumor response and histologic evaluation of BISIC}

Objective tumor responses on PM and primary tumors were evaluated by CT and gastroesophageal endoscopy before and after BISC. Histologic effects on primary tumors and PM were evaluated according to the general rules for gastric cancer treatment [6]. Histological response after chemotherapy is classified into 4 categories. EF-0 shows no histologic response or response less than one third of the tumor tissue. A histologic EF-1 means that the degeneration of cancer is detected in the tumor tissue raging from one third to less than two thirds of the tumor tissue. EF-2 shows the degeneration of cancer tissue in wider than two thirds of the tumor tissue, while an EF-3 means the complete disappearance of the cancer cells.

\section{Evaluation of toxicity}

Patients were evaluated to assess the extent of disease, before entry into the study and after 3 cycles of BISIC, by physical examination, Computed tomography (CT), upper gastrointestinal series, endoscopic examination and peritoneal cytologic status. Blood cell count, liver and renal function test, electrolytes and urinalysis were done once every two weeks. The response of primary tumors and PM were evaluated after 3 cycles of this treatment according to the response criteria of the Japanese Research Society for Gastric Cancer [6]. The National Cancer Institute Common Toxicity Criteria (NCI-CTC) version 3 was applied to evaluate adverse drug reactions. Postoperatie complication was assessed based on Common Terminology Criteria for Adverse Events v 4.0.

The primary end point was 1-year Overall survival (OS) rate.
Secondary end points were the overall response rate (ORR), histological effects and safety.

\section{Statistical analyses}

All patients were followed and no patients were lost to followup. Outcome data were obtained from medical records and patients' interview. All statistical analyses were performed using SPSS software statistical computer package version 17 (SPSS Inc., Chicago, USA).

\section{Results}

From June 2012 to December 2013, 61 patients were enrolled in this study (Table 1). Only 47 patients with measurable target lesions were assessed for Overall response rate (ORR). The ORR was 29\% with 14 patients showing partial response. In 33 patients with primary tumor, 29 patients $(88 \%)$ showed partial response.

The chemotherapy was discontinued due to adverse events in 2 patients, and due to disease progression in 15 patients. In 44 patients, CRS was done 3 to 4 weeks after the last cycle of chemotherapy.

Before BISIC, cytology had been positive in $38(67.8 \%)$ of 57 patients. These 38 positive cytological results before BISIC became negative in 27 (71.1\%) patients after BISIC (Table 2).

After BISIC, 44 patients received laparotomy and CRS. At laparotomy, mean Peritoneal cancer index (PCI) [7] was 8.5, ranging from 0 to 27.

Total gastrectomy was performed in 25 patients. A variety of supplemental procedures were performed to achieve complete

Table 1. Patient's characteristics.

\begin{tabular}{|l|c|}
\hline Sex & \\
\hline Male & 39 \\
\hline Female & 32 \\
\hline Age, years & 54 \\
\hline Median & $31-75$ \\
\hline Range & \\
\hline ECOG performance status & 45 \\
\hline 0 & 16 \\
\hline 1 & \\
\hline Primary or recurrence & 38 \\
\hline Primary & 23 \\
\hline Recurrence & \\
\hline Histologic type & 4 \\
\hline Differentiated & 57 \\
\hline Poorly differentiated & 16 \\
\hline Macroscopic type & 45 \\
\hline Type 3 & \\
\hline Type 4 & 44 \\
\hline Treatments & 17 \\
\hline CRS\# after BISIC\& & \\
\hline BISC alone & \\
\hline
\end{tabular}

\# CRS: Cytoreductive Surgery

\& BISIC: Bidirectional Intraperitoneal Systemic Induction Chemotherapy

Table 2. Peritoneal cytological status before and after BISIC

\begin{tabular}{|c|c|c|c|}
\hline & \multicolumn{2}{|c|}{ Three cycles after BISIC } & \\
\hline Before BISIC & negative & positive & \\
\hline negative & 18 & 1 & $\mathbf{1 9}$ \\
\hline positive & 27 & 11 & $\mathbf{3 8}$ \\
\hline & $\mathbf{4 5}$ & $\mathbf{1 2}$ & 57 \\
\hline
\end{tabular}


Yutaka Yonemura (2014) A new bidirectional intraperitoneal and systemic induction chemotherapy (BISIC) for the peritoneal metastasis from gastric cancer in neoadjuvant setting

Table 3. Numbers of patients with toxic effects during BISIC.
\begin{tabular}{|l|c|c|c|c|c|}
\hline Toxicity & \multicolumn{5}{|c|}{ Grade (CTCAE v.3.0) } \\
\hline & $\mathbf{1}$ & $\mathbf{2}$ & $\mathbf{3}$ & $\mathbf{4}$ & $\mathbf{5}$ \\
\hline Leukopenia & 1 & 1 & 3 & & \\
\hline Fatigue & 2 & 4 & 1 & 1 & \\
\hline Diarrhea & 5 & & & & \\
\hline Mucositis & & 2 & & & \\
\hline Meningitis & & & & 1 & \\
\hline Port infection & & & & 1 & \\
\hline
\end{tabular}

Table 4. Mortality and morbidity after CRS

\begin{tabular}{|l|c|c|c|c|c|}
\hline & \multicolumn{5}{|c|}{ Grade } \\
\hline Bleeding & $\mathbf{1}$ & $\mathbf{2}$ & $\mathbf{3}$ & $\mathbf{4}$ & $\mathbf{5}$ \\
\hline Intestinal fistula & & & & 2 & 1 \\
\hline Leakage & & & & 1 & \\
\hline Pancreatic fistula & & & & & 1 \\
\hline Wouddehiciency & & & 2 & & \\
\hline Abscess & & & 1 & & \\
\hline
\end{tabular}

Table 5. Histological effects after BISIC.

\begin{tabular}{|l|l|l|l|l|}
\hline Lesions & EF-0 & EF-1 & EF-2 & EF-3 \\
\hline Primary tumors (N=33) & $4(12.1 \%)$ & $19(57.6 \%)$ & $9(27.3 \%)$ & $1(4.0 \%)$ \\
\hline Peritoneal metastasis (N=44) & $6(13.6 \%)$ & $19(43.2 \%)$ & $9(20.5 \%)$ & $10(22.7 \%)$ \\
\hline
\end{tabular}

cytoreduction. Total colectomy, right hemicolectomy, left hemicolectomy and transverse colectomy were done in $9,8,2$, and 2 patients, respectively. Colectomies combined with low anterior resection were done in 10 patients. Segmental resection of small bowel and small bowel mesentery was done in 24 patients. Hysterectomy combined with bilateral salpingo-oophorectomy was performed in 23 patients. For parietal peritonectomy, pelvic peritonectomy, subdiaphragmatic peritonectomy and peritonectomy of para-colic gutter were done in 31, 19 and 44 patients. Mean operation time was $227 \mathrm{~min}$ (120 380 $\mathrm{min}$ ), and mean blood loss was $1344 \mathrm{ml}(100 \sim 3500 \mathrm{ml})$.

Complete cytoreduction (CC-0) was achieved in 28 (64\%) of 44 patients. During BISIC, side effects of grade 3 and 4 were found in 4 $(6.6 \%)$ and $2(3.3 \%)$ patients (Table 3 ). The frequent grade $3 / 4$ toxic effects included leucopenia (4.9\%) and fatigue (3.3\%). After CRS, 4 (9.0\%) and $3(6.8 \%)$ patients developed Grade 3 and 4 complications (Table 4). The most frequent complications are bleeding in 3 . The overall operative mortality rate was $4.5 \%(2 / 44)$, and the cause of death was multiple organ failure due to leakage and bleeding. Grade 4 complications were found in 3 patients, and 3 patients underwent operation for the postoperative bleeding in two patient and bowel fistula in one patient.

Histologic effects on primary tumors were found in 29 (87.9\%) of the 33 tumors, and EF-1, $-2,-3$ response in the primary tumors were detected in 19 (57.6\%), 9 (27.3\%) and 1 tumor (4.0\%), respectively (Table 5). Complete histologic disappearance of PM was observed in $10(22.7 \%)$ of 44 patients (Table 5). Thirty-six patients were alive at the time of analysis. The survival curve for all patients is shown in Figure 1. Median Survival Time (MST) was 12.2 months, with a one and twoyear survival of $62.4 \%$, and $44.0 \%$.

\section{Discussion}

After the comprehensive treatment for PM, complete cytoreduction is the strongest prognostic factor for the long-term survival [79]. However, survival of patients with PCI score higher than the threshold value is poor, even if they received complete cytoreduction. Diagnostic laparoscopy showed that complete ctroreduction was supposed to achieve only in fewer than $30 \%$ of gastric cancer-patients with PM who had not been treated with neoadjuvant chemotherapy [10]. Accordingly, PCI levels higher than the threshold value should be reduced within the threshold level by Neoadjuvant chemotherapy (NAC) before CRS,

Systemic chemotherapy is usually used as NAC. However, the effects of systemic chemotherapy on PM are limited, and no longterm survivors were reported after systemic chemotherapy. Takeyoshi et al. reported the median overall survival by weekly paclitaxel with doxifluridine was 215 days, and 1-year survival rate was only $29.2 \%$ [11]. The reason is considered that the peritoneal cavity acts as a sanctuary against systemic chemotherapy, because of the existence of a Blood-peritoneal barrier (BPB). BPB consists of stromal tissue between mesothelial cells and submesothelial blood capillaries [12]. Accordingly, only a small amount of systemic drugs are capable of penetrating this barrier.

In contrast, i.p. chemotherapy offers potential therapeutic advantages over systemic chemotherapy by generating very high locoregional intensity of drugs in the peritoneal cavity [13]. Coccolini F et al. reported that i.p. chemotherapy+CRS is associated with improved overall survival [14].

For the i.p. chemotherapy, degree recommended drugs which stay long time in the peritoneal cavity after i.p. administration [15]. Molecular weights of paclitaxel, docetaxel, gemcitabine, 5-fluorouracil and doxorubicinis are high. After i,p, administration of these drugs, the ratio of the area under the drug concentration-time curve (AUC) in the peritoneal cavity and AUC in plasma (AUCp/AUCs) is higher than those after i,p, administration of other drugs [15]. Among these drugs, AUCp/AUCs were much larger for paclitaxel and docetaxel than for other drugs [16]. They are administered as micellar preparation, Taxol and Taxotere, which consists of paclitaxel in Cremophor and docetaxel in Polysorbate-80. Paclitaxel and docetaxel are slowly released from micellar compounds, and the relatively higher intraperitoneal concentration of the paclitaxel and docetaxel are maintained for a long time [16]. From these results, Yonemura et al. developed Neoadjuvant intraperitoneal/systemic chemotherapy (NIPS) [17]. NIPS can eradicate PFCCs before CRS, and may prevent the attachment of PFCCs on the surgical wound at CRS. In addition, complete histologic response on PM was found in $37 \%$ of patients after NIPS [17].

More recently, alternate administration of systemic and intraperitoneal chemotherapy was developed [18] and we designated the method Bidirectional Intraperitoneal and Systemic Induction Chemotherapy (BISIC). BISIC creates a wider treatment area than

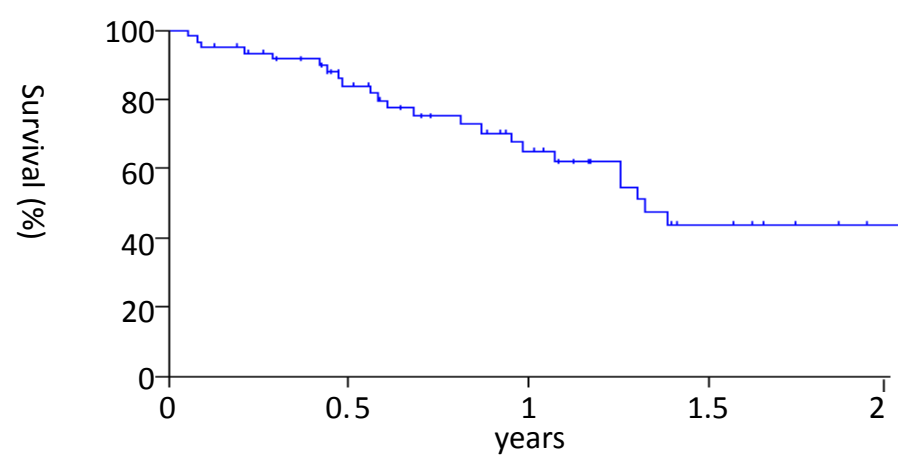

Figure 1. Overall survival of patients treated with BISIC 
Yutaka Yonemura (2014) A new bidirectional intraperitoneal and systemic induction chemotherapy (BISIC) for the peritoneal metastasis from gastric cancer in neoadjuvant setting

single treatment by a bidirectional diffusion gradient. In the present study, positive cytology before BIPSC became negative in $79 \%$ of patients after 3 cycles of BISIC. Histologic response rates on PM after BISIC and NIPS were $86.4 \%(38 / 44)$ and $60 \%$ (88/147), respectively [17] and the histologic response rate after BISIC was significantly higher than that after NIPS. In addition, one-year survival of patients after BISC and NIPS were $62 \%$ and $36 \%$ [17]. These results may indicate that BISIC has more effective for survival than NIPS. An analysis of longterm survival after BISC is awaited.

During BISIC, side effects of grade 3 and 4 were found in $9.9 \%$ $(6 / 61)$ among 61 patients. The frequent grade $3 / 4$ toxic effects included leucopenia (4.9\%) and fatigue (3.3\%). All patients recovered after appropriate infusion therapy. Ishigami et al. developed a new BISIC consists of weekly i.v. and i.p. paclitaxel combined with S1 [18]. However, grade $3 / 4$ toxic effects were found in $38 \%$ of patients, including neutropenia (38\%) and anemia (10\%), and were higher than our results.

These results indicate that NIPS and BISIC are effective therapies for the eradication of PFCCs and for the reduction of PCI score. BISIC has more powerful in histologic response on PM than NIPS.

Accordingly, BISIC considered being a safe method as compared with the results of previous reports of systemic chemotherapy. However, BISIC might increase the mortality rate after CRS. After CRS, $4(9.0 \%)$ and $3(6.8 \%)$ patients developed Grade 3 and 4 complications, and mortality was experienced in $4.5 \%(2 / 44)$ of patients.

Glehen reported a higher complication rate of $47 \%$ in patients who underwent extensive cytoreductive surgery (gastrectomy combined with the removal of more than 2 peritoneal zones) [19]. The magnitude of surgery, the number of resected organs, the number of anastomosis, and the operation time are considered to have contributed to the significantly higher complication rate. To avoid futile aggressive treatments, the preoperative and intraoperative stringent selection of patients must be emphasized.

\section{References}

1. Hong AH, Shin YR, Roh Y, Jeon EK, Song KY, et al. (2013) Treatment outcomes of systemic chemotherapy for peritoneal carcinomatosis arising from gastric cancer with no measurable disease: retrospective analysis from a single center. Gastric Cancer 16: 290-300. [Crossref]

2. Glehen O, Yonemura Y, Sugarbaker H (2013) Cytoreductive surgery \& periopertaive chemotherapy for peritoneal surface malignancy. Chapter 4; Prevention and treatment of peritoneal metastases from gastric cancer. P79-89. Textbook and Video Atlas. Ed. Paul Sugarbaker PH, Cine-Med Publishing, Inc., North Woodburry, CT, USA

3. Yonemura Y, Canbay E, Endou Y, Ishibashi H, Mizumoto A, et al. (2014) Peritoneal cancer treatment. Expert Opin Pharmacother 15: 623-636. [Crossref]
4. Yonemura Y, Bandou E, Sawa T, Yoshimitsu Y, Endou Y, et al. (2006) Neoadjuvant treatment of gastric cancer with peritoneal dissemination. Eur J Surg Oncol 32: 661665. [Crossref]

5. Yonemura Y, Elnemr A, Endou Y, Hirano M, Mizumoto A, et al. (2010) Multidisciplinary therapy for treatment of patients with peritoneal carcinomatosis from gastric cancer World J Gastrointest Oncol 2: 85-97. [Crossref]

6. Japanese Research Society for Gastric Cancer (1995) The General Rules for Gastric Cancer Study ( $1^{\text {st }}$ English ed). Tokyo. KaneharaShuppan.

7. Jacquet P, Sugarbaker PH (1996) Clinical research methodologies in diagnosis and staging of patients with peritoneal carcinomatosis. Cancer Treat Res 82: 359-374. [Crossref]

8. Sugarbaker PH (1999) Successful management of microscopic residual disease in large bowel cancer. Cancer Chemother Pharmacol 43: S15-25. [Crossref]

9. Yan TD, Morris DL (2008) Cytoreductive surgery and perioperative intraperitonea chemotherapy for isolated colorectal peritoneal carcinomatosis: experimental therapy or standard of care? Ann Surg 248: 829-835. [Crossref]

10. Valle M, Van der SpeetenGalafalo (2009) Laparoscopic hyperthermic intraperitoneal preoperative chemotherapy (HIPEC) in the management of refractory malignant ascites: A multi-institutional retrospective analysis in 52 patients. $J$ Surg Oncol 15:331334

11. Takeyoshi I, Makita F, Iwazaki S, Ishikawa H, Kakinuma S, et al. (2011) Weekly paclitaxel in combination with doxifluridine for peritoneally disseminated gastric cancer with malignant ascites. Anticancer Res 31: 4625-4630. [Crossref]

12. Baron MA (1941) Structure of intestinal peritoneum in man. Am J Anat 69:439-497.

13. Markman M (1991) Intraperitoneal therapy in ovarian cancer utilizing agents acjieving high local but low systemic exposure. Reg Cancer Treat 40:256-260.

14. Coccolini F, Cotte E, Glehen O, Lotti M, Poiasina E, et al. (2014) Intraperitonea chemotherapy in advanced gastric cancer. Meta-analysis of randomized trials. Eur J Surg Oncol 40: 12-26. [Crossref]

15. deBree E, Tsiftsis DD (2007) Experimental and pharmacologic studies in intraperitonea chemotherapy from laboratory bench to bedside. Advances in peritoneal surface oncology. S. Gonzalez-Moleno ed. Springer: 53-73.

16. Miyamoto K, Shimada T, Sawamoto K, Sai Y, Yonemura Y (2012) Disposition kinetics of taxanes in peritoneal dissemination. Gastroenterol Res Pract 2012: 963403. [Crossref]

17. Yonemura Y (2012) Effects of Neoadjuvant Intraperitoneal/Systemic Chemotherapy (Bidirectional Chemotherapy) for the Treatment of Patients with Peritoneal Metastasis from Gastric Cancer. International Journal of Surgical Oncology. [Crossref]

18. Ishigami H, Kitayama J, Kaisaki S, Hidemura A, Kato M, et al. (2010) Phase II study of weekly intravenous and intraperitoneal paclitaxel combined with S-1 for advanced gastric cancer with peritoneal metastasis. Ann Oncol 21: 67-70. [Crossref]

19. Glehen O, Gilly FN, Arvieux C, Cotte E, Boutitie F, et al. (2010) Peritoneal carcinomatosis from gastric cancer; A multi-institutional study of 159 patients treated by cytoreductive surgery combined with perioperative intraperitoneal chemotherapy. Ann Surg Oncol 17: 2370-2307. [Crossref]

Copyright: (C2014 Yonemura Y. This is an open-access article distributed under the terms of the Creative Commons Attribution License, which permits unrestricted use, distribution, and reproduction in any medium, provided the original author and source are credited. 